\title{
Article \\ MicroRNAs as a Suitable Biomarker to Detect the Effects of Long-Term Exposures to Nanomaterials. Studies on $\mathrm{TiO}_{2} \mathrm{NP}$ and MWCNT
}

\author{
Sandra Ballesteros ${ }^{1}$, Gerard Vales ${ }^{2}$, Antonia Velázquez ${ }^{1}$, Susana Pastor ${ }^{1}$, Mohamed Alaraby ${ }^{1,3}$, Ricard Marcos ${ }^{1, *}$ \\ and Alba Hernández ${ }^{1, *}$ \\ 1 Group of Mutagenesis, Department of Genetics and Microbiology, Faculty of Biosciences, \\ Universitat Autònoma de Barcelona, 08193 Cerdanyola del Vallès, Spain; sandra.ballesteros@uab.es (S.B.); \\ antonia.velazquez@uab.es (A.V.); susana.pastor@uab.es (S.P.); \\ mohamed.alaraby@science.sohag.edu.eg (M.A.) \\ 2 Finnish Institute of Occupational Health, 00250 Helsinki, Finland; gerard.vales@ttl.fi \\ 3 Zoology Department, Faculty of Sciences, Sohag University, Sohag 82524, Egypt \\ * Correspondence: ricard.marcos@uab.cat (R.M.); alba.hernandez@uab.cat (A.H.)
}

check for updates

Citation: Ballesteros, S.; Vales, G.; Velázquez, A.; Pastor, S.; Alaraby, M.; Marcos, R.; Hernández, A.

MicroRNAs as a Suitable Biomarker to Detect the Effects of Long-Term Exposures to Nanomaterials. Studies on $\mathrm{TiO}_{2} \mathrm{NP}$ and MWCNT.

Nanomaterials 2021, 11, 3458. https:// doi.org/10.3390/nano11123458

Academic Editor: Zhiyong Zhang

Received: 22 October 2021

Accepted: 17 December 2021

Published: 20 December 2021

Publisher's Note: MDPI stays neutral with regard to jurisdictional claims in published maps and institutional affiliations.

Copyright: (c) 2021 by the authors. Licensee MDPI, Basel, Switzerland. This article is an open access article distributed under the terms and conditions of the Creative Commons Attribution (CC BY) license (https:// creativecommons.org/licenses/by/ $4.0 /)$.

\begin{abstract}
The presence of nanomaterials (NMs) in the environment may represent a serious risk to human health, especially in a scenario of chronic exposure. To evaluate the potential relationship between NM-induced epigenetic alterations and carcinogenesis, the present study analyzed a panel of 33 miRNAs related to the cell transformation process in BEAS-2B cells transformed by $\mathrm{TiO}_{2} \mathrm{NP}_{\text {and }}$ long-term MWCNT exposure. Our battery revealed a large impact on miRNA expression profiling in cells exposed to both NMs. From this analysis, a small set of five miRNAs (miR-23a, miR-25, miR-96, miR-210, and miR-502) were identified as informative biomarkers of the transforming effects induced by NM exposures. The usefulness of this reduced miRNA battery was further validated in other previously generated transformed cell systems by long-term exposure to other NMs (CoNP, ZnONP, $\mathrm{MSiNP}$, and $\mathrm{CeO}_{2} \mathrm{NP}$ ). Interestingly, the five selected miRNAs were consistently overexpressed in all cell lines and NMs tested. These results confirm the suitability of the proposed set of mRNAs to identify the potential transforming ability of NMs. Particular attention should be paid to the epigenome and especially to miRNAs for hazard assessment of NMs, as wells as for the study of the underlying mechanisms of action.
\end{abstract}

Keywords: microRNAs; oncogenesis; carcinogenesis; cell transformation; nanomaterials; long-term exposures; $\mathrm{TiO}_{2} \mathrm{NP} ; \mathrm{MWCNT}$

\section{Introduction}

The nanotechnology industry is constantly producing new nanomaterials (NMs) with potential applicability in many fields. Consequently, their presence in our environment has substantially increased in recent years [1]. Due to their high surface-to-volume ratio, NMs show high biological reactivity when interacting with different cellular molecules and therefore have the potential to induce adverse effects on any exposed organism, including humans [2]. Accordingly, it is necessary to investigate the potential toxic, genotoxic, and cumulative effects of NMs, as well as to develop new biomarkers for the correct assessment of their possible negative effects on both humans and the environment [3].

Although many experimental models and biological endpoints have been used to evaluate the harmful effects of NMs, few studies have evaluated their potential carcinogenic risks using long-term in vitro approaches. Since carcinogenesis is a complex, multi-step process, prolonged exposures over time are necessary to induce cell transformation. Hence, a long-term exposure approach is required when evaluating the carcinogenic potential of a given compound. However, such an experimental exposure scenario is not frequently 
found in NM testing studies since most studies focus on short-term in vitro exposures using high doses of the compound.

It has already been demonstrated that long-term in vitro exposures to non-cytotoxic doses of NMs are able to induce the acquisition of different hallmarks of cancer in exposed cells, such as morphological cell changes, secretion of matrix metalloproteinases, anchorageindependent cell-growth capacity, and migration ability, as observed after exposures to cobalt nanoparticles [4], zinc oxide nanoparticles [5], silver nanoparticles [6], and multiwalled carbon nanotubes (MWCNT) [7]. On the other hand, emerging evidence indicates that NM exposure can cause different epigenetic changes correlated with gene-expression alterations [8-11]. Among them, changes in the level of microRNA (miRNA) expression have been associated with different pathological processes [12,13]. Interestingly, different studies have demonstrated that many cancers have alternative miRNA expression profiles when they are compared to normal tissues. This indicates that initiation, proliferation, and control of the apoptotic program of tumor cells are modulated by different epigenetic entities, including miRNAs [14]. In this direction, we have recently shown that following long-term exposure to nanoceria, bronchial epithelial BEAS-2B cells acquire an oncogenic phenotype characterized by an increased cell-invasion capacity and tumorsphere-formation ability [15]. Interestingly, we demonstrated that these oncogenic changes were accompanied by altered expression of several miRNAs with known roles in the carcinogenesis process [15]. More specifically, expression levels of about the 30\% of an initial panel of 33 candidates showed relevant changes, which included miRNAs considered oncogenes (OncomiRs) or tumor suppressors (Anti-OncomiRs), based on whether they target tumor suppressor genes or oncogenes, respectively [16]. From that work, we proposed that the panel of miRNAs could be used as biomarker for the evaluation of the carcinogenic potential of NMs.

Here, to reinforce our proposal and further explore the connection between the acquisition of the oncogenic phenotype and the altered miRNA profile, we evaluated the expression levels of the 33 miRNAs from our panel in BEAS-2B cells following long-term exposure to titanium dioxide nanoparticles $\left(\mathrm{TiO}_{2} \mathrm{NP}\right)$ or MWCNT for 6 weeks. After exposure to both NMs, BEAS-2B cells exhibited an oncogenic phenotype according to the results obtained in a soft-agar assay. This assay measures anchorage-independent cell-growth capacity, one of the main characteristics of transformed cells [17,18]. BEAS-2B cells were selected as the target since inhalation is the most likely route of unintentional human exposure to different NMs, including $\mathrm{TiO}_{2} \mathrm{NP}$ and MWCNT. Both NMs have been extensively used, and their environmental consequences and potential effects on humans have been recently reviewed $[19,20]$.

This study moves one step further than the previous one [15] by identifying a reduced set of informative miRNAs useful in detection of potential oncogenic effects induced by NM exposures. Since miRNAs participate in many overlapping cellular processes, the miRNA-level changes of the proposed set can also be conceived as more general biomarkers for toxicological assessment of NMs under scenarios of long-term exposure. Interestingly, this set of miRNAs was successfully validated in other long-term exposed cell systems previously transformed by cobalt nanoparticles (CoNP), zinc oxide nanoparticles (ZnONP), mesoporous silica nanoparticles (MSiNP), and cerium dioxide nanoparticles $\left(\mathrm{CeO}_{2} \mathrm{NP}\right)$.

\section{Materials and Methods}

\subsection{Nanomaterials Characterization}

$\mathrm{TiO}_{2} \mathrm{NP}$ (NM-102) and MWCNT (NM-401) were obtained from the repository of the EU Joint Research Centre (Ispra, Italy) in the frame of the EU NanoReg project. Further characterization of these materials was performed using transmission electron microscopy (TEM, JEOL JEM-2011 instrument) (Jeol LTD, Tokyo, Japan).) to determine dry size and morphology. The mean sizes were determined by measuring 100 randomly selected nanoparticles in several TEM images using the ImageJ program. Specifically, the mean size of MWCNT was calculated by measuring the width of isolated fibers. Dynamic light scattering (DLS), 
and laser Doppler velocimetry (LDV) methodologies (Malvern Zetasizer Nano-ZS zen3600 instrument) (Malvern, UK). were used to determine hydrodynamic size and zeta potential, respectively. For NMs dispersion, $\mathrm{TiO}_{2} \mathrm{NP}$ and $\mathrm{MWCNT}$ were pre-wetted in $0.5 \%$ ethanol and dispersed in $0.05 \%$ bovine serum albumin (BSA) in Milli-Q water. Afterward, the NMs were sonicated for $16 \mathrm{~min}$ to obtain a stock dispersion of $2.56 \mathrm{mg} / \mathrm{mL}$, according to the NanoGenotox protocol [21]. For TEM and Zetasizer measurements, the stock suspension was dispersed in water and in culture medium, respectively.

\subsection{Cell Culture and Exposure Conditions}

BEAS-2B cells were cultured as a monolayer in T75 flasks with DMEM medium (Gibco, Paisley, UK) supplemented with 10\% fetal bovine serum (FBS; Pasching, Austria), 1\% nonessential amino acids (Pasching, Austria), and $2.5 \mu \mathrm{g} / \mathrm{mL}$ Plasmocin (InvivoGen, San Diego, CA, USA) and incubated at $37{ }^{\circ} \mathrm{C}$ in a humidified atmosphere of $5 \% \mathrm{CO}_{2}$. During the 6-week duration of the long-term exposure, sub-confluent cells were passaged weekly at a cell density of $5 \times 10^{5}$ per T75 flask, and the medium was changed every two days by removing the old medium, then washing the cells twice with phosphate-buffered saline (PBS), then adding new fresh medium containing the NM exposure. Exposures consisted of non-cytotoxic concentrations of 10 and $20 \mu \mathrm{g} / \mathrm{mL}$ of $\mathrm{TiO}_{2} \mathrm{NP}$ and MWCNT, equivalent to 1.34 and $2.67 \mu \mathrm{g} / \mathrm{cm}^{2}$. Importantly, non-treated, time-matched controls were maintained in parallel during the complete period of exposure. All conditions were performed in triplicates for each of the three experiments carried out.

To determine the toxicity of the long-term exposures, a viability assay was performed using the Beckman counter method (Beckman Coulter, Brea, CA, USA). The average number of viable cells for each exposure time point was compared to time-matched, nonexposed controls to calculate cell viability percentages.

\subsection{RNA Extraction and Retrotranscription Experiments}

Changes in the miRNA level of expression were evaluated by real-time RT-PCR. To proceed, total RNA was isolated from long-term exposed and time-matched controls = BEAS-2B cells using TRI Reagent ${ }^{\circledR}$ (Invitrogen, Waltham, MA, USA), in triplicate. RNA quantity was measured on a Nanodrop spectrophotometer (Thermo Fisher Scientific Technologies,

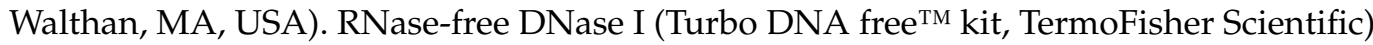
was used to remove DNA contamination. An amount of $80 \mathrm{ng}$ of total RNA in a final volume of $10 \mu \mathrm{L}$ was used for cDNA synthesis. These $10 \mu \mathrm{L}$ included $1 \mu \mathrm{L}$ of 10X poly(A) polymerase buffer, $10 \mathrm{mM}$ of ATP, $1 \mu \mathrm{M}$ of RT-primer (Sigma-Aldrich, Steinheim, Germany), $0.1 \mathrm{mM}$ of each deoxynucleotide (dATP, dCTP, dGTP, and dTTP) (VWR International, Ballicoolin, Dublin, Ireland), 100 units of MulV reverse transcriptase (New England Biolabs, Ipswich, MA, USA), and 1 unit of poly(A) polymerase (New England, Biolabs, Ipswich, MA, USA). The mix was incubated at $37^{\circ} \mathrm{C}$ for $1 \mathrm{~h}$, followed by enzyme inactivation at 95 ${ }^{\circ} \mathrm{C}$ for $5 \mathrm{~min}$. The sequence of the RT-primer was $5^{\prime}$-CAGGTCCAGTTTTTTTTTTTTTTTVN, where $\mathrm{V}$ is $\mathrm{A}, \mathrm{C}$, and $\mathrm{G}$ and $\mathrm{N}$ is $\mathrm{A}, \mathrm{C}, \mathrm{G}$, and $\mathrm{T}$.

\subsection{Real-Time RT-PCR}

cDNA was amplified by RT-qPCR on a LightCycler480. Quantitative PCR was performed in $10 \mu \mathrm{L}$ of total volume with $3 \mu \mathrm{L}$ of cDNA, $5 \mu \mathrm{L}$ of $2 X$ LightCycler 480 SYBR Green I Master (Roche, Mannheim, Germany), $250 \mathrm{nM}$ of each primer (Sigma-Aldrich) (see Table 1), and $1 \mu \mathrm{L}$ of $\mathrm{H}_{2} \mathrm{O}$. The primers were designed using miRprimer software [22,23]. Cycling conditions were $95^{\circ} \mathrm{C}$ for $1 \mathrm{~min}$, followed by 55 cycles of $95^{\circ} \mathrm{C}$ for $1 \mathrm{~min}$ and $65^{\circ} \mathrm{C}$ for $30 \mathrm{~s}$. Cycle threshold $(\mathrm{Ct})$ values were calculated with the Lightcycler software package and then normalized with U6 values. Experiments were performed in triplicate. Statistical analysis was performed by the $2^{-\Delta \Delta \mathrm{C}_{\mathrm{T}}}$ method to compare exposed cells with untreated controls. In all cases, a two-sided $p<0.05$ was considered statistically significant. 
Table 1. MicroRNA primers sequences.

\begin{tabular}{|c|c|c|}
\hline & FORWARD & REVERSE \\
\hline >hsa-miR-21-5p & TCAGTAGCTTATCAGACTGATG & CGTCCAGTTTTTTTTTTTTTTTCAAC \\
\hline$>$ hsa-miR-23a-5p & CATCACATTGCCAGGGAT & CGTCCAGTTTTTTTTTTTTTTTGGAA \\
\hline >hsa-miR-25-3p & CATTGCACTTGTCTCGGT & GGTCCAGTTTTTTTTTTTTTTTCAG \\
\hline$>$ hsa-miR-30c-5p & GCAGTGTAAACATCCTACACTCT & TCCAGTTTTTTTTTTTTTTTGCTGA \\
\hline$>$ hsa-miR-30d-5p & AGTGTAAACATCCCCGACT & GGTCCAGTTTTTTTTTTTTTTTCTTC \\
\hline >hsa-miR-31-5p & GCAGAGGCAAGATGCTG & GTCCAGTTTTTTTTTTTTTTTAGCTATG \\
\hline >hsa-miR-33a-5p & CGCAGGTGCATTGTAGT & GTCCAGTTTTTTTTTTTTTTTGCAAT \\
\hline$>$ hsa-miR-34a-5p & GCAGTGGCAGTGTCTTAG & GGTCCAGTTTTTTTTTTTTTTTACAAC \\
\hline >hsa-miR-96-5p & CAGTTTGGCACTAGCACA & GGTCCAGTTTTTTTTTTTTTTTAGCA \\
\hline$>$ hsa-miR-124-5p & GCAGCGTGTTCACAGC & TCCAGTTTTTTTTTTTTTTTATCAAGGT \\
\hline >hsa-miR-126-5p & CGCAGCATTATTACTTTTGGT & CCAGTTTTTTTTTTTTTTTCGCGT \\
\hline >hsa-miR-132-5p & ACCGTGGCTTTCGATTG & GGTCCAGTTTTTTTTTTTTTTTAGTAAC \\
\hline$>$ hsa-miR-135b-5p & GCAGTATGGCTTTTCATTCCT & GGTCCAGTTTTTTTTTTTTTTTCACA \\
\hline$>$ hsa-miR-148b-3p & GCAGTCAGTGCATCACAGA & GGTCCAGTTTTTTTTTTTTTTTACAAAG \\
\hline$>$ hsa-miR-154-5p & GCAGTAGGTTATCCGTGTTG & GTCCAGTTTTTTTTTTTTTTTCGAAG \\
\hline >hsa-miR-155-5p & CGCAGTTAATGCTAATCGTGATAG & AGGTCCAGTTTTTTTTTTTTTTTACC \\
\hline$>$ hsa-miR-199b-5p & CAGCCCAGTGTTTAGACTATC & GTCCAGTTTTTTTTTTTTTTTGAACAG \\
\hline >hsa-miR-200a & AGCATCTTACCGGACAGT & CCAGTTTTTTTTTTTTTTTCCAGCA \\
\hline$>$ hsa-miR-200b-5p & GCATCTTACTGGGCAGCA & GGTCCAGTTTTTTTTTTTTTTTCCAA \\
\hline >hsa-miR-200c & CGTCTTACCCAGCAGTGT & GGTCCAGTTTTTTTTTTTTTTTCCA \\
\hline$>$ hsa-miR-203a-3p & CAGGTGAAATGTTTAGGACCA & GGTCCAGTTTTTTTTTTTTTTTCTAGT \\
\hline >hsa-miR-210-5p & TGCCCACCGCACA & GGTCCAGTTTTTTTTTTTTTTTCAGT \\
\hline$>$ hsa-miR-218-5p & CGCAGTTGTGCTTGATCT & TCCAGTTTTTTTTTTTTTTTACATGGT \\
\hline$>$ hsa-miR-222-3p & GCAGAGCTACATCTGGCT & CCAGTTTTTTTTTTTTTTTACCCAGT \\
\hline >hsa-miR-224-5p & GCAGCAAGTCACTAGTGGT & TCCAGTTTTTTTTTTTTTTTAACGGA \\
\hline >hsa-miR-342-3p & GTCTCACACAGAAATCGCA & GGTCCAGTTTTTTTTTTTTTTTACG \\
\hline$>$ hsa-miR-486-3p & GGGGCAGCTCAGTACA & GGTCCAGTTTTTTTTTTTTTTTATCCT \\
\hline >hsa-miR-502-3p & AATGCACCTGGGCAAG & GGTCCAGTTTTTTTTTTTTTTTGAATC \\
\hline$>$ hsa-miR-505-3p & CGTCAACACTTGCTGGT & GGTCCAGTTTTTTTTTTTTTTTAGGA \\
\hline >hsa-miR-541-3p & GTGGTGGGCACAGAATC & CCAGTTTTTTTTTTTTTTTAGTCCAG \\
\hline hsa-miR-544a & GCAGATTCTGCATTTTTAGCAAG & GGTCCAGTTTTTTTTTTTTTTTGAAC \\
\hline >hsa-miR-939-3p & CCTGGGCCTCTGCTC & GGTCCAGTTTTTTTTTTTTTTTCTG \\
\hline$>$ hsa-miR-1271-3p & TGCCTGCTATGTGCCA & TCCAGTTTTTTTTTTTTTTTGCCT \\
\hline >U6 small nuclear RNA & CTCGCTTCGGCAGCACA & AACGCTTCACGAATTTGCGT \\
\hline
\end{tabular}

\subsection{Pathway-Enrichment Analysis}

The significantly deregulated miRNAs in each of the evaluated conditions were imported to the miRTarBase 8.0 database. Common target genes were selected and imported to the DAVID database to perform a pathway-enrichment analysis. Pathways with a $p$-value $<0.05$ and a fold enrichment higher than 10 were selected.

\subsection{Validation of the Selected Battery of MicroRNAs as Biomarkers of Long-Term Effects of NMs}

A subset of five miRNAs (miR-23a, miR-25, miR-96, miR-210 and miR-502) was found to be sufficient to identify the effects induced by $\mathrm{TiO}_{2} \mathrm{NP}$ and long-term $\mathrm{MWCNT}$ exposure. To confirm its suitability, its performance was tested with different NMs and cellular backgrounds. Thus, mouse embryonic fibroblast knockouts for the Ogg1 gene (MEF Ogg1-/- cells; gift from Dr. Deborah Barnes at Cancer Research UK, UK) previously exposed for 12 weeks to $1 \mu \mathrm{g} / \mathrm{mL}$ of zinc oxide nanoparticles $(\mathrm{ZnONP})$ and to $0.1 \mu \mathrm{g} / \mathrm{mL}$ of cobalt nanoparticles (CoNP) were used [4,5]. In addition, BEAS-2B cells previously exposed for 6 weeks to mesoporous silica nanoparticles (MSiNP) at $10 \mu \mathrm{g} / \mathrm{mL}$ (unpublished results) and cerium dioxide nanoparticles $\left(\mathrm{CeO}_{2} \mathrm{NP}\right)$ at $2.5 \mu \mathrm{g} / \mathrm{mL}$ [15] were included. Longterm cell culture conditions and miRNA analysis were conducted, as reported in the previous sections. 


\section{Results}

To determine the characteristics of the used NMs, TEM and DLS methodologies were applied. Figure $1 \mathrm{~A}$ show representative TEM images of $\mathrm{TiO}_{2} \mathrm{NP}$ and MWCNT. While MWCNTs present the fibbers-like shape characteristic of nanotubes, $\mathrm{TiO}_{2} \mathrm{NPs}$ show a more spherical size with irregularities. The mean sizes obtained from TEM images are shown in Figure 1B. The values of hydrodynamic radius and zeta potential are also indicated in Figure 1B. The differences in size observed between TEM and DLS for $\mathrm{TiO}_{2} \mathrm{NP}$ are indicative of a certain degree of aggregation shown by this nanomaterial, as observed in TEM figures and in the polydispersion index value $(0.4 \pm 0.1)$. In addition, the Z-potential value for $\mathrm{TiO}_{2} \mathrm{NP}(-25.4 \pm 0.1 \mathrm{mV})$ also suggests moderate colloidal stability and the ability to resist aggregation. Similarly, the results obtained for MWCNT also indicate a certain degree of aggregation.

A.
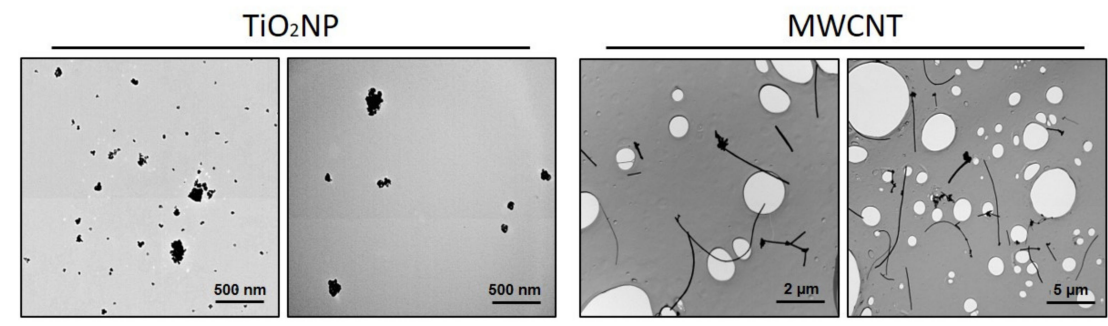

B.

\begin{tabular}{|lcc|}
\hline & $\mathrm{TiO}_{2} \mathrm{NP}$ & MWCNT \\
\hline Size $(\mathrm{nm})(\mathrm{TEM})$ & $20.1 \pm 7.4$ & $5.9 \pm 4.6$ \\
\hline Size $(\mathrm{nm})(\mathrm{DLS})$ & $612.4 \pm 44.8$ & $882.1 \pm 2.7$ \\
\hline Pdl (DLS) & $0.4 \pm 0.1$ & $0.6 \pm 0.2$ \\
\hline Z-potential (mV) (DLV) & $-25.4 \pm 0.1$ & $22.8 \pm 0.8$ \\
\hline Mobility $(\mu \mathrm{m} \mathrm{cm} / \mathrm{Vs})(\mathrm{DLV})$ & $2.0 \pm 0.1$ & $1.8 \pm 0.1$ \\
\hline
\end{tabular}

Figure 1. Characterization of $\mathrm{TiO}_{2} \mathrm{NP}$ and MWCNT. (A) TEM representative images of both NPs. (B) Different parameters, as measures by TEM and Zetasizer. Data are represented as mean \pm SD $(n=3)$.

The concentrations selected for $\mathrm{TiO}_{2} \mathrm{NP}$ and MWCNT were based on previous toxicity studies, where they did not exert significant effects on cell viability [18,19]. To confirm that $\mathrm{TiO}_{2} \mathrm{NP}$ and MWCNT were not toxic at the tested concentration in our long-term exposure model, the viability of the exposed cells at week 3 and week 6 was assessed, and the obtained results (Figure 2) indicate that there was no significant decrease in cell viability associated with the treatments or exposure times.
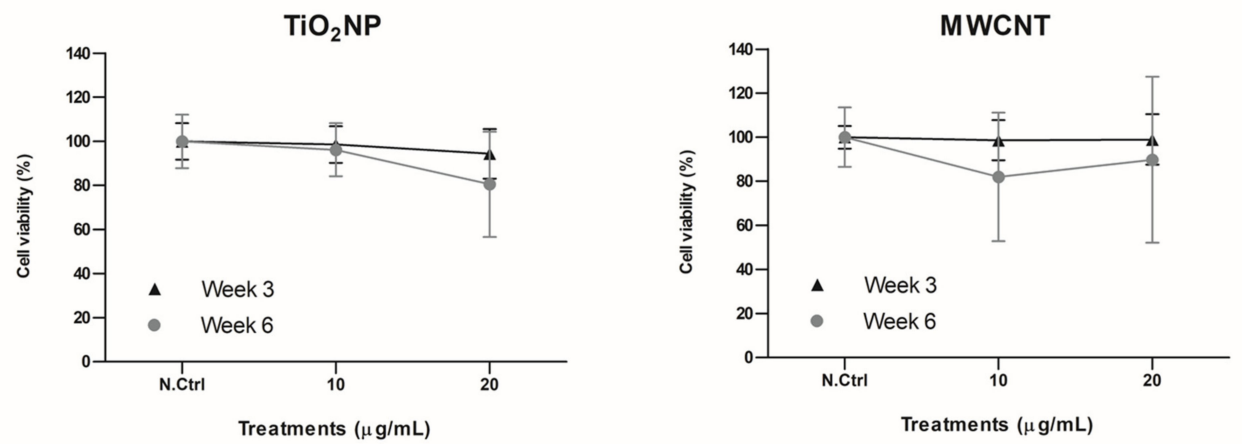

Figure 2. Relative viability of BEAS-2B cells at weeks 3 and 6 of chronic exposure to $\mathrm{TiO}_{2} \mathrm{NP}$ and MWCNT. Viability percentages were calculated by averaging the number of cells counted for each condition in three independent experiments. Cell viability is represented as the percentage of counted cells compared to the untreated time-matched controls \pm SEM. Data were analyzed by comparing each condition to the untreated time-matched control (Student's $t$-test). 


\subsection{MicroRNA Expression Changes after $\mathrm{TiO}_{2} \mathrm{NP}$ Exposure}

Figure 3 shows the analysis of the observed response after $\mathrm{TiO}_{2} \mathrm{NP}$ exposure at the highest concentration used. The expression analysis at the lowest concentration is shown in Supplementary Figure S1. Among the entire panel of miRNAs, $29 \%$ were significantly deregulated at both tested time points: $41.9 \%$ at week 3 and 29\% at week 6 .

A.

\section{$\mathrm{TiO}_{2} \mathrm{NP}$}

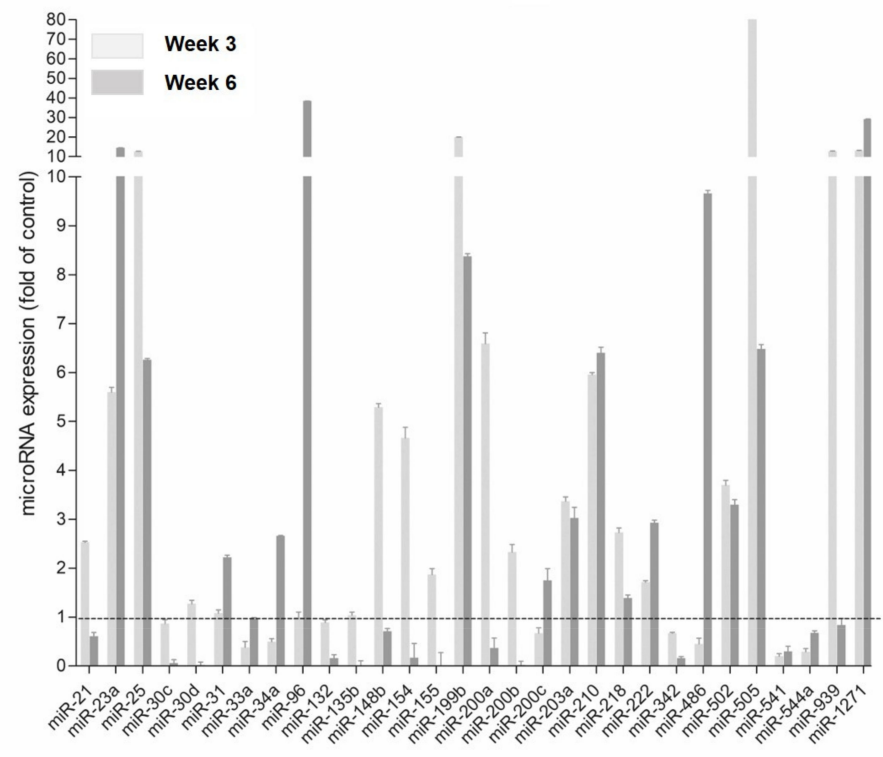

B.

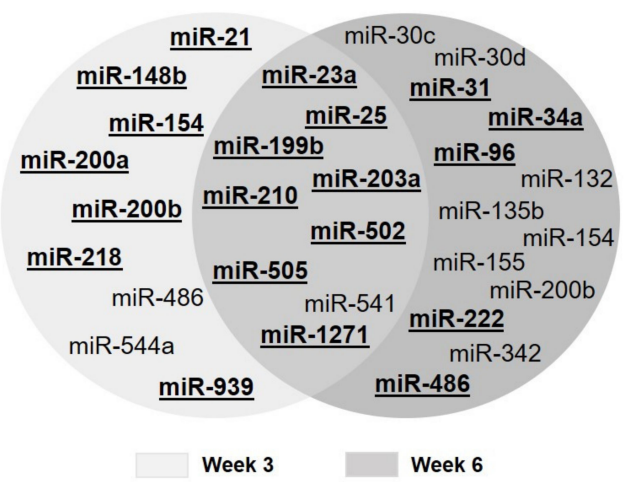

Figure 3. MiRNA expression changes of BEAS-2B cells exposed to the highest concentration $(20 \mu \mathrm{g} / \mathrm{mL})$ of $\mathrm{TiO}_{2} \mathrm{NP}$. (A) Deregulated miRNAs at week 3 and week 6 after exposure to $\mathrm{TiO}_{2} \mathrm{NP}$. Data are plotted as mean, and error bars represent the SEM. (B) Venn diagram showing the number of miRNAs significantly deregulated at week 3 and week 6 of exposure. The overlapping area indicates the number of miRNAs commonly deregulated at both exposure times. Overexpressed miRNAs are in bold and underlined. Results were analyzed with the Student's $t$-test $(p<0.05)$.

Among those deregulated at both time-points, most miRNAs showed an overexpression compared to the time-matched controls. The six most upregulated miRNAs were miR-23a, miR-25, miR-199b, miR-210, miR-505, and miR-1271. For instance, miR-505 and miR-1271 showed overexpression of $86.21 \pm 0.26$ and $13.10 \pm 0.20$ folds at week 3 and $6.48 \pm 0.23$ and $29.15 \pm 0.27$ folds at week 6 , respectively. On the contrary, only miR-541 appeared downregulated at both weeks [0.2 \pm 0.13 week $3 ; 0.3 \pm 0.25$ week 6]. Among those miRNAs showing significant changes only at week 6 (Figure 3B), miR-96 was the most upregulated (38.43 \pm 0.13 folds). As for week 3, miR-148b, miR-200a, and miR-939 
were found to be highly upregulated. In addition, there were some miRNAs showing different behaviors, depending on the evaluated sampling point (week). Thus, while miR-154 and miR-200b were overexpressed at week 3 and downregulated at week 6, miR-486 was inhibited at week 3 but underwent a significant increase in expression at week 6 .

\subsection{MicroRNA Expression Changes after MWCNT Exposure}

Those miRNAs deregulated after exposure to the highest concentration of MWCNT are represented in Figure 4A. The expression analysis at the lowest concentration is shown in Supplementary Figure S2. Figure 4B represents those miRNAs that were significantly deregulated at week 3 , at week 6 , or at both exposure times.

A.

\section{MWCNT}

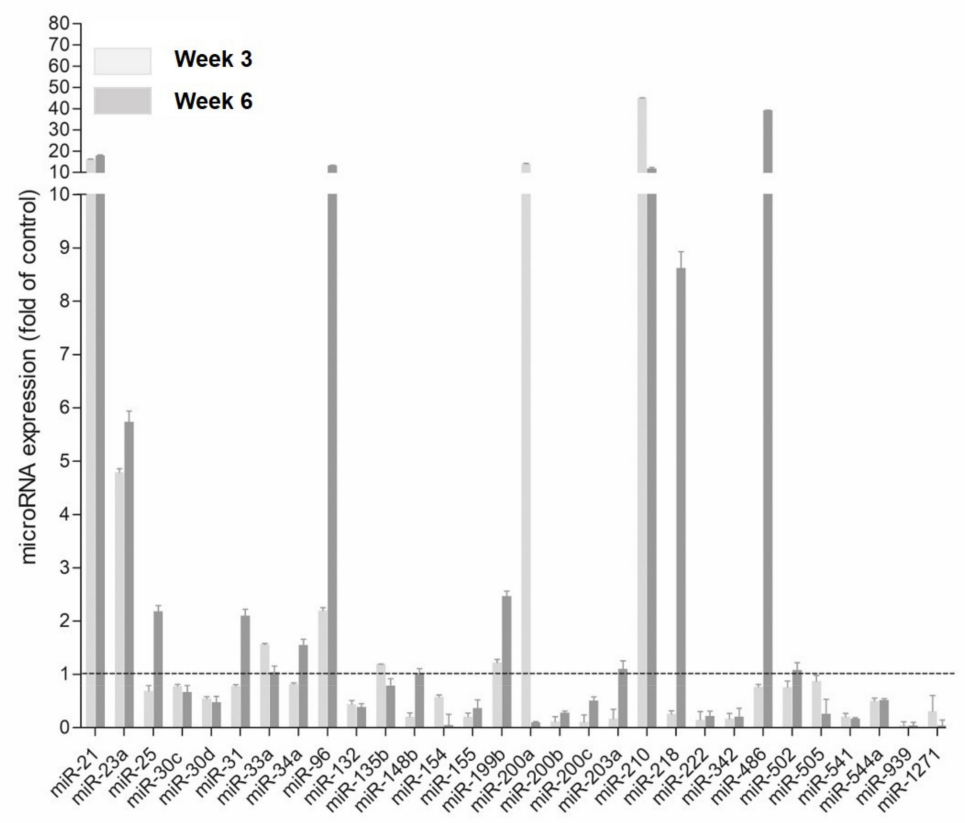

B.

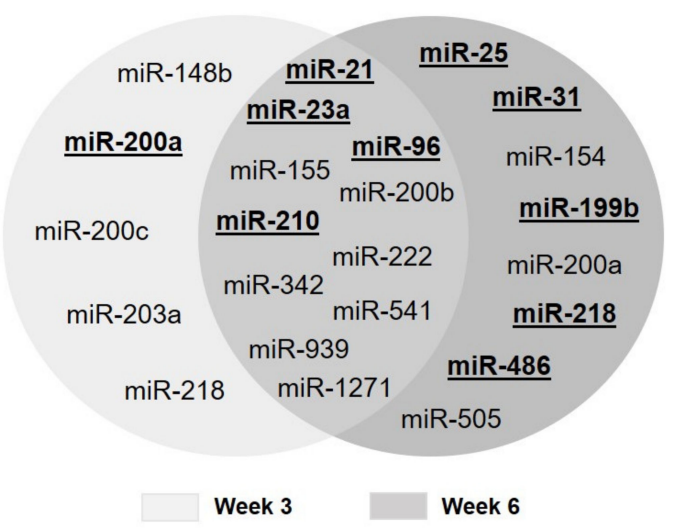

Figure 4. MiRNA expression changes of BEAS-2B cells exposed to the highest concentration $(20 \mu \mathrm{g} / \mathrm{mL}$ ) of MWCNT. (A) Deregulated miRNAs at week 3 and week 6 after the exposure to MWCNT. Data are plotted as mean, and error bars represent the SEM. (B) Venn diagram showing the number of miRNAs significantly deregulated at week 3 and week 6 of exposure. The overlapping area indicates the number of miRNAs commonly deregulated at both exposure times. Overexpressed miRNAs are in bold and underlined. Results were analyzed with the Student's $t$-test $(p<0.05)$. 
Most miRNAs were deregulated in both stages of the exposure, with only miR-21, miR23a, miR-96, and miR-210 being overexpressed. From them, miR-21 and miR-210 reached an overexpression of at least $16.23 \pm 0.16$ and $45 \pm 0.43$ folds at week 3 and $18.07 \pm 0.53$ and $11.87 \pm 0.99$ folds at week 6 , respectively. However, most of them presented underexpression at both times (miR-155, miR-200b, miR-222, miR-342, miR-541, miR-939, and miR-1271). Among those that were inhibited, miR-541 and miR-939 showed an expression of $0.21 \pm 0.14$ and $0.04 \pm 0.17$ folds at week 3 and $0.17 \pm 0.04$ and $0.04 \pm 0.15$ folds at week 6 , respectively. On the other hand, miR-25, miR-31, miR-154, miR-199b, miR-200a, miR-218, miR-486, and miR-505 were only deregulated at week 6 of exposure. Among them, miR-154, miR-200a, and miR-505 were the only ones downregulated. On the contrary, at week 3 , most were downregulated (miR-148b, miR-200c, miR-203a, and miR-218), except for miR200a. This miRNA showed a different expression behavior, depending on the observed exposure time; thus, while at early stages, it was upregulated, it was under-expressed at the late stages. The opposite happened with miR-218, which was downregulated at week 3 and upregulated at week 6 .

\subsection{Pathway-Enrichment Analysis}

Figure 5 represents those functional pathways enriched when DAVID analysis was carried out. This analysis was performed based on the potential common target genes from the miRTarBase of those miRNAs significantly deregulated at week 3 and week 6 after the exposure to $\mathrm{TiO}_{2} \mathrm{NP}(\mathrm{A})$ and MWCNT (B). The enriched pathways after $\mathrm{TiO}_{2} \mathrm{NP}$ exposure (week 3) were: (i) cell-cycle arrest and (ii) signal transduction (NIK/NF-kappaB, FGF, and TGF $\beta$ signaling pathways). On the other hand, the most significantly enriched pathways at week 6 were (i) cell-death programs (senescence, apoptosis, autophagy), (ii) cell-cycle arrest, (iii) heat-shock response, (iv) inflammatory response, (v) cell migration, and (vi) signal transduction (growth factor and interferon-gamma-signaling pathways). Regarding enriched pathways after MWCNT exposure at week 3, they were (i) cell-cycle arrest, (ii) signal transduction (Wnt and epidermal-growth-factor-signaling pathways), (iii) heat-shock response, and (iv) adherent junctions. Finally, those enriched at week 6 were (i) cell-cycle arrest, (ii) cellular senescence, (iii) angiogenesis, (iv) cell migration, and (v) signal transduction (TGF $\beta$ signaling pathway).

\section{(A) $\mathrm{TiO}_{2} \mathrm{NP}$}

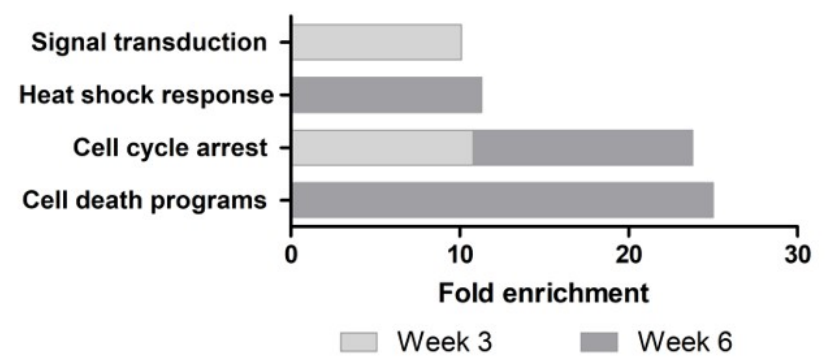

\section{(B) MWCNT}

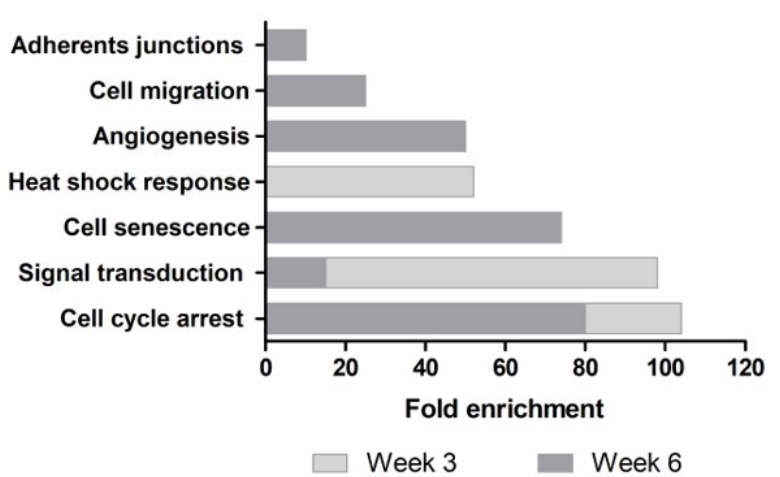

Figure 5. Pathway-enrichment analysis for the target genes of the miRNAs significantly deregulated at week 3 and week 6 after the exposure to (A,B). Pathways were selected based on $p$-value $<0.05$ and fold enrichment higher than 10 . 


\subsection{Validation of a Set of MicroRNAs as Informative Biomarkers of Long-Term Effects of NMs}

From the analysis of the results obtained in the expression of our initial battery of miRNAs, we propose a small set of miRNAs as potential biomarkers of effect after exposure to NMs. Thus, miR-23a, miR-96, and miR-210 were initially included because they were overexpressed in all concentrations and times of the exposure to both NMs $\left(\mathrm{TiO}_{2} \mathrm{NP}\right.$ and MWCNT). In addition, miR-25 and miR-502 were added to the list because they were overexpressed in all concentrations and times of at least one NM-in our case, after $\mathrm{TiO}_{2} \mathrm{NP}$ exposure. To validate the usefulness of the five proposed miRNAs, we checked their response in other cells following previous long-term exposure to other NMs, namely CoNP, $\mathrm{ZnONP}$, MSiNP, and $\mathrm{CeO}_{2} \mathrm{NP}$. The obtained results are indicated in Figure 6 . As observed, all five candidate miRNAs showed significant overexpression in all tested conditions, i.e., CoNP exposure the condition inducing the greater response.

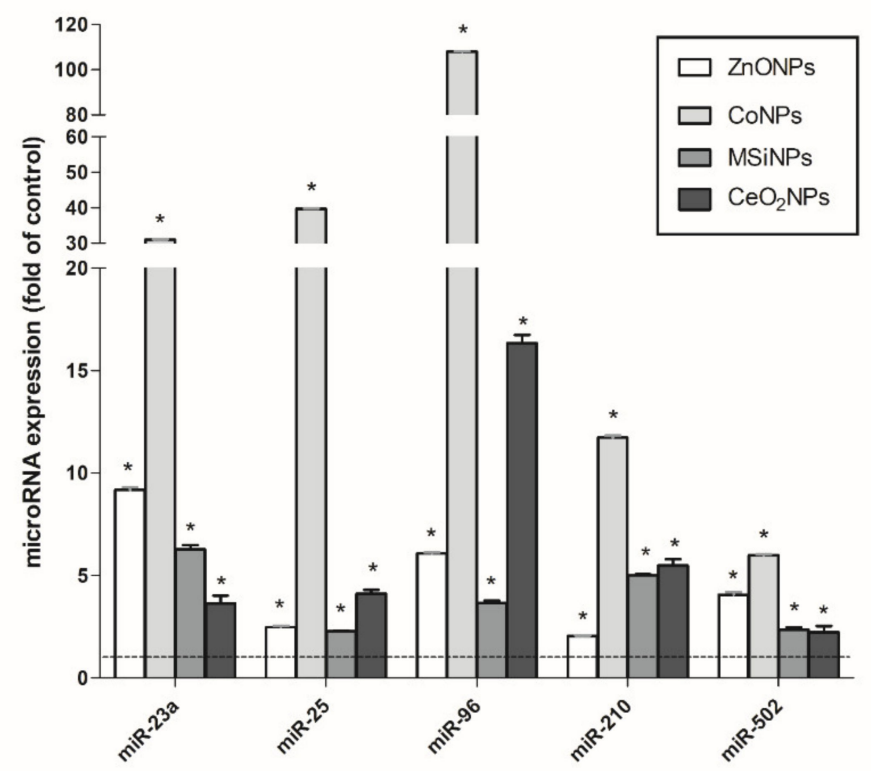

Figure 6. microRNA expression changes in the set of selected miRNAs (miR-23a, miR-25, miR-96, miR-210, miR-502) after the exposure to ZnONP, CoNP, MSiNP, and $\mathrm{CeO}_{2} \mathrm{NP}$. Data are plotted as mean, and error bars represent the SEM. Results were analyzed with the Student's $t$-test $\left({ }^{*} p<0.05\right)$.

Finally, a pathway-enrichment analysis was performed with the common target genes of this selected/proposed set of miRNAs. The three most enriched pathways were (i) signal transduction (PI3k, IGF-1, Wnt, mTOR, and FoxO-signaling pathways), (ii) adherent junctions, and (iii) cell-cycle arrest (Figure 7). Thus, most miRNAs from the selected set of miRNAs act as controlling genes from the three functional categories. However, two of the selected miRNAs (miR-23a and miR-502) were only present in the (i) signal-transduction and (ii) cell-cycle-arrest categories. Additionally, each pathway is controlled by several target genes, and the overlapping area among the three pathways indicates that EP300 controls the three pathways. At the same time, each one of the genes is controlled by several miRNAs, as indicated by the colored bar below the target gene. 


\section{Signal transduction Adherent junctions}

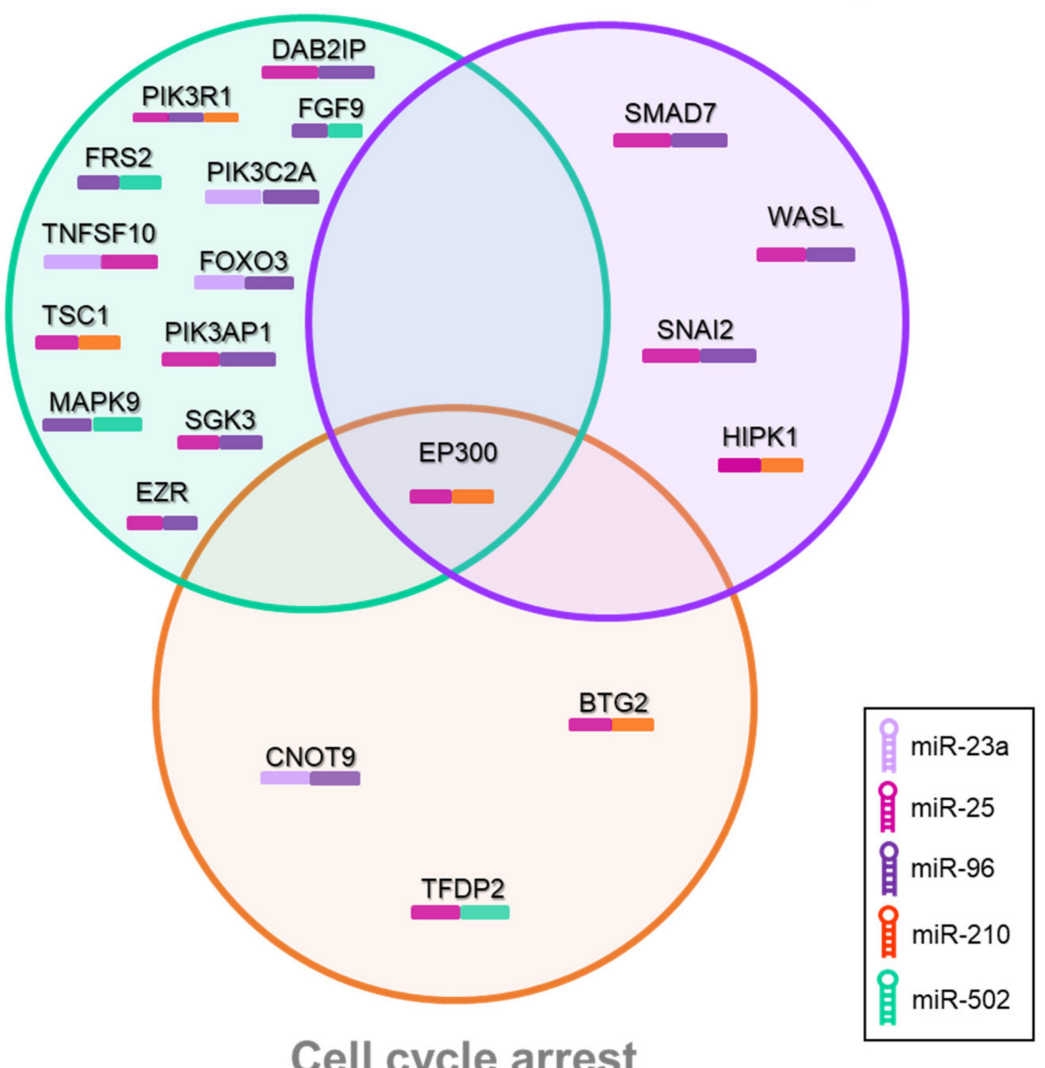

Figure 7. Venn diagram showing the pathway-enrichment analysis for the common target genes of the set of selected miRNAs (miR-23a, miR-25, miR-96, miR-210, miR-502). Signal transduction, adherent junctions, and cell-cycle arrest were the most enriched pathways. Colored bars under target genes represent the miRNAs of the legend. Pathways were selected based on $p$-value $<0.05$ and fold enrichment higher than 10.

\section{Discussion}

At present, enough pieces of evidence demonstrate that exposure to NMs can lead to a wide set of harmful effects, including inflammatory response, DNA damage, oxidative stress, lipid peroxidation, apoptosis, altered gene expression, immunotoxicity, reproductive toxicity, and carcinogenesis [24,25]. Most of the studies generating such information have been conducted using in vitro methods as suitable approaches to in vivo-induced effects. However, most of these studies are far away from simulating real human exposures scenarios since they use short-term exposures and high (and non-biologically relevant) concentrations. Therefore, there is a need to implement in vitro chronic-exposure models in the field and to use them to assay low and non-cytotoxic/concentrations of the NMs under study [26]. An additional advantage of in vitro long-term exposure systems is that they permit the detection of different long-term effects, such as those related to the acquisition of a tumoral phenotype. Indeed, the cell-transformation ability of different NMs has already been reported using these experimental approaches, as demonstrated with cobalt nanoparticles [4], titanium dioxide nanoparticles [19], multiwalled carbon nanotubes [20], nickel oxide nanoparticles [26], and cerium oxide [15].

Epigenetic changes are among the different biomarkers associated with cell transformation processes [11], including miRNA expression changes [27]. In the present study, BEAS-2B cells following long-term exposure to $\mathrm{TiO}_{2} \mathrm{NP}$ and MWCNT showed altered expression changes in a battery of 33 miRNAs related to inflammation, cellular stress, or the carcinogenesis processes. Thus, our system revealed a large impact on miRNA 
expression in cells exposed to both $\mathrm{NMs} \mathrm{TiO}_{2} \mathrm{NP}$ exposure triggered overexpression of miR-21, miR-148b, miR-154, miR-200a, miR-200b, miR-218, and miR-939 at the early stages of the exposure. These miRNAs target genes implicated in cell-cycle arrest, signal transduction, and adherent junctions. It is worth highlighting that miR-21 targets some common tumor-suppressor genes, such as PTEN or PDCD4 [28]. The PTEN gene plays a key role in regulation of the cell cycle, inhibiting cell growth and division at the protein level [29]. Moreover, an elevated expression of miR-21 has been related to poor prognosis in many types of cancers [30]. After 6 weeks of exposure, a large number of miRNAs appeared to be downregulated (miR-30c, miR-30d, miR-132, miR-135b, miR-154, miR-155, miR-200b, and miR-342), while others were overexpressed (miR-31, miR-34a, miR-96, and miR-132). Interestingly, these miRNAs control pathways related to cell-death programs, cell-cycle arrest, heat-shock response, inflammatory response, cell migration, and signal transduction.

Regarding the effects observed under long-term exposures to MWCNT, miR-148b, miR200c, miR-203a, and miR-218 showed under-expression at week 3 . They all mostly control pathways related to cell-cycle arrest, signal transduction, heat-shock response, and adherent junctions. After 6 weeks of exposure, most of the miRNAs were observed to be upregulated, such as miR-25, miR-31, miR-199b, miR-218, miR-486, while miR-154, miR-200a, and miR505 were found to be downregulated. All of them were related to cell-cycle arrest pathways, cell senescence, angiogenesis, cell migration, and signal-transduction pathways.

One of the conclusions reached from the analysis of the effects of this wide battery of 33 miRNAs is that a few of them can be representative of the epigenetic alterations induced by nanomaterials. Accordingly, five miRNAs, namely miR-23a, miR-25, miR-96, miR-210, and miR-502, were proposed to be used as a set of biomarkers of effect when the health consequences of different nanomaterials are evaluated. Among them, and according to the literature, miR-23a potentiates the epithelial-to-mesenchymal transition process (EMT), downregulating E-cadherin and increasing expression of vimentin, two key proteins in the EMT process [31]. Similarly, another study found that miR-23a was overexpressed in exosomes derived from A549 human lung adenocarcinoma cells, inducing EMT [32]. Moreover, this process has been related to metastasis and poor prognosis in lung cancer patients [33]. Regarding miR-25, its upregulation was correlated with lymph node metastasis, with a poor prognosis in non-small-cell lung cancer (NSCLC) patients [34].

Regarding the role of specific miRNAs in the regulation of the tumoral process, they can act as oncomiRs or as tumor-suppressor miRs. According to the literature, the five miRNAs selected in our study act as oncomiRs [35,36]. The overexpression of miR-96 after all exposures correlates with studies that evidence its role as oncomiR. These studies demonstrate that the overexpression of miR-96 promotes proliferation and invasion and inhibits apoptosis in NSCLC by targeting different genes, such as LMO7, RECK, and FOXO3 [37-39]. Additionally, it is known that miRNAs can interchange their roles as oncomiRs or tumor-suppressor miRs, according to the tumor type. They can simultaneously produce promoting and tumor-suppressive effects. The balance between their effects will determine whether a specific miRNA produces a net oncogenic or net tumor-suppressive effect. At present, there is conflicting literature as to whether specific miRNAs are oncogenic or tumor-suppressive [40]. As an example, miR-1271 interchanges its role between either oncomiR or tumor suppressor miR, depending on the tumor type and even depending on the target that we observe within the same type of cancer. Similar behavior has been recently described for miR-1297 [41]. From our results, this miRNA exhibits different behavior, depending on the exposure. While $\mathrm{TiO}_{2} \mathrm{NP}$ exposure triggered its overexpression, MWCNT exposure inhibits its expression.

Interestingly, the goodness of the selected five miRNAs as constituents of a battery useful for evaluation of the cell-transforming potential of NMs was confirmed when applied to transformed cells resulting from previous experiments. These BEAS-2B/MEF cells come from long-term cultures exposed to different nanomaterials (CoNP, ZnONP, $\mathrm{MSiNP}$, and $\mathrm{CeO}_{2} \mathrm{NP}$ ) and expressing different biomarkers of cell transformation. 


\section{Conclusions}

To conclude, our study evidences that long-term, low-dose exposure to NMs induces miRNA expression changes directly associated with the oncogenic phenotype. Although our data cannot clearly state whether these miRNAs suppose "the spark" needed to start a tumoral process or whether they are symptoms of the tumoral phenotype, we consider that this area is an interesting field requiring further investigation. It should be emphasized that since miRNAs participate in many overlapping cellular processes, the miRNA-level changes of the proposed set can also be conceived of as more general biomarkers for toxicological assessment of NMs under long-term scenarios of exposure. Thus, understanding the link between miRNA expression changes and the long-term effectsinduced by NMs exposure, such as carcinogenesis, can provide valuable information about the underlying mechanism(s), which will benefit NM hazard and risk assessment. To this end, the different pathways identified to be key targets of the proposed set of miRNAs can be considered a promising starting point. In this way, our proposal of using a restricted set of miRNAs can be a powerful tool to determine the potential carcinogenic risk of environmental exposures to NMs. Obviously, the use of this battery can be extended to any agent suspected to have carcinogenic potential.

Exposure to many types of environmental agents has been reported to be able to deregulate the expression of different miRNAs, and such regulation can be used as a biomarker of cancer development induced by environmental factors [42]. Among such environmental factors, plastics and plasticizers are among the most interesting emergent pollutants. In this context, a recent revision indicates that plasticizers alter the expression of different miRNAs. Such a genotoxic/oncogenic response could eventually lead to alterations in the cell signaling pathways involved in different overlapping cellular processes [43]. As a consequence, they can be useful targets in the assessment of the harmful effects of environmental agents, including nanomaterials.

Supplementary Materials: The following are available online at https:/ / www.mdpi.com/article/ 10.3390/nano11123458/s1, Figure S1: MicroRNA expression changes of BEAS-2B cells exposed to the lowest concentration of TiO2NPs. Figure S2: MicroRNA expression changes of BEAS-2B cells exposed to the lowest concentration of MWCNTs.

Author Contributions: R.M. and A.H. planned the experiments; S.B., G.V., A.V., S.P. and M.A. carried out the experimental part; S.B. analyzed the data, carried out the statistical analysis and prepared tables/figures; S.B., A.H. and R.M. wrote the final manuscript. All authors have read and agreed to the published version of the manuscript.

Funding: This work was partially supported by the Spanish Ministry of Science and Innovation [PID2020-116789, RB-C43]. Sandra Ballesteros holds a fellowship from the Universitat Autonoma de Barcelona [PIF-UAB].

Institutional Review Board Statement: Not applicable.

Informed Consent Statement: Not applicable.

Data Availability Statement: Not applicable.

Acknowledgments: We wish to thank C. Valiente for his technical assistance.

Conflicts of Interest: The authors declare no conflict of interest.

Competing Financial Interests: The authors declare they have no actual or potential competing financial interests.

\section{References}

1. Bimová, P.; Barbieriková, Z.; Grenčíková, A.; Šípoš, R.; Škulcová, A.B.; Krivjanská, A.; Mackul'ak, T. Environmental risk of nanomaterials and nanoparticles and EPR technique as an effective tool to study them-a review. Environ. Sci. Pollut. Res. Int. 2021, 28, 22203-22220. [CrossRef] [PubMed]

2. Tian, X.; Chong, Y.; Ge, C. Understanding the nano-bio interactions and the corresponding biological responses. Front. Chem. 2020, 8, 446. [CrossRef] [PubMed] 
3. Freixa, A.; Acuña, V.; Sanchís, J.; Farré, M.; Barceló, D.; Sabater, S. Ecotoxicological effects of carbon based nanomaterials in aquatic organisms. Sci. Total Environ. 2018, 619-620, 328-337. [CrossRef] [PubMed]

4. Annangi, B.; Bach, J.; Vales, G.; Rubio, L.; Marcos, R.; Hernández, A. Long-term exposures to low doses of cobalt nanoparticles induce cell transformation enhanced by oxidative damage. Nanotoxicology 2015, 9, 138-147. [CrossRef]

5. Annangi, B.; Rubio, L.; Alaraby, M.; Bach, J.; Marcos, R.; Hernández, A. Acute and long-term in vitro effects of zinc oxide nanoparticles. Arch. Toxicol. 2016, 90, 2201-2213. [CrossRef]

6. Vila, L.; Marcos, R.; Hernández, A. Long-term effects of silver nanoparticles in Caco-2 cells. Nanotoxicology 2017, 11, 771-780. [CrossRef]

7. Barthel, H.; Darne, C.; Gaté, L.; Visvikis, A.; Seidel, C. Continuous long-term exposure to low concentrations of MWCNTs induces an epithelial-mesenchymal transition in BEAS-2B cells. Nanomaterials 2021, 11, 1742. [CrossRef]

8. Sierra, M.I.; Rubio, L.; Bayón, G.F.; Cobo, I.; Menendez, P.; Morales, P.; Mangas, C.; Urdinguio, R.G.; Lopez, V.; Valdes, A.; et al. DNA methylation changes in human lung epithelia cells exposed to multi-walled carbon nanotubes. Nanotoxicology 2017, 11, 857-870. [CrossRef]

9. Smolkova, B.; Dusinska, M.; Gabelova, A. Nanomedicine and epigenome. Possible health risks. Food Chem. Toxicol. 2017, 109, 780-796. [CrossRef]

10. Yu, J.; Loh, X.J.; Luo, Y.; Ge, S.; Fan, X.; Ruan, J. Insights into the epigenetic effects of nanomaterials on cells. Biomater. Sci. 2020, 8, 763-775. [CrossRef]

11. Pogribna, M.; Hammons, G. Epigenetic effects of nanomaterials and nanoparticles. J. Nanobiotechnol. 2021, 19, 2. [CrossRef] [PubMed]

12. Paul, P.; Chakraborty, A.; Sarkar, D.; Langthasa, M.; Rahman, M.; Bari, M.; Singha, R.K.S.; Malakar, A.K.; Chakraborty, S. Interplay between miRNAs and human diseases. J. Cell. Physiol. 2018, 233, 2007-2018. [CrossRef]

13. Huang, Q.; Liu, Y.; Dong, S. Emerging roles of long non-coding RNAs in the toxicology of environmental chemicals. J. Appl. Toxicol. 2018, 38, 934-943. [CrossRef] [PubMed]

14. Chekhun, V.F. MicroRNAs are a key factor in the globalization of tumor-host relationships. Exp. Oncol. 2019, 41, 188-194. [CrossRef] [PubMed]

15. Ballesteros, S.; Barguilla, I.; Marcos, R.; Hernández, A. Ex vivo long-term in vitro exposures to nanoceria, alone or in combination with cigarette-smoke condensate, induce transforming and epigenetic cancer-like features. Nanomedicine 2021, 16, 293-305. [CrossRef]

16. George, G.P.; Mittal, R.D. MicroRNAs: Potential biomarkers in cancer. Ind. J. Clin. Biochem. 2010, 25, 4-14. [CrossRef] [PubMed]

17. Vales, G.; Rubio, L.; Marcos, R. Long-term exposures to low doses of titanium dioxide nanoparticles induce cell transformation, but not genotoxic damage in BEAS-2B cells. Nanotoxicology 2015, 9, 568-578. [CrossRef]

18. Vales, G.; Rubio, L.; Marcos, R. Genotoxic and cell-transformation effects of multi-walled carbon nanotubes (MWCNT) following in vitro sub-chronic exposures. J. Hazard. Mater. 2016, 306, 193-202. [CrossRef]

19. Shabbir, S.; Kulyar, M.F.; Bhutta, Z.A.; Boruah, P.; Asif, M. Toxicological consequences of titanium dioxide nanoparticles (TiO $\left.{ }_{2} \mathrm{NPs}\right)$ and their jeopardy to human population. BioNanoScience 2021, 26, 621-632. [CrossRef]

20. Saleemi, M.A.; Hosseini, F.M.; Yong, P.V.C.; Chinna, K.; Palanisamy, N.K.; Wong, E.H. Toxicity of carbon nanotubes: Molecular mechanisms, signaling cascades, and remedies in biomedical applications. Chem. Res. Toxicol. 2021, 34, 24-46. [CrossRef] [PubMed]

21. Nanogenotox. 2011. Available online: https://www.anses.fr/en/system/files/nanogenotox_deliverable_5.pdf (accessed on 2 November 2021).

22. Balcells, I.; Cirera, S.; Busk, P.K. Specific and sensitive quantitative RT-PCR of miRNAs with DNA primers. BMC Biotechnol. 2011, 11, 70. [CrossRef]

23. Busk, P.K. A tool for design of primers for microRNA-specific quantitative RT-qPCR. BMC Bioinform. 2014, 15, 29. [CrossRef]

24. Khanna, P.; Ong, C.; Bay, B.; Baeg, G. Nanotoxicity: An interplay of oxidative stress, inflammation and cell death. Nanomaterials 2015, 5, 1163-1180. [CrossRef] [PubMed]

25. Ramanathan, A. Toxicity of nanoparticles challenges and opportunities. Appl. Microsc. 2019, 49, 2. [CrossRef] [PubMed]

26. Gliga, A.R.; Bucchianico, S.D.; Åkerlund, E.; Karlsson, H.L. Transcriptome profiling and toxicity following long-term, low dose exposure of human lung cells to $\mathrm{Ni}$ and $\mathrm{NiO}$ nanoparticles-comparison with $\mathrm{NiCl}_{2}$. Nanomaterials 2020, 10, 649. [CrossRef]

27. Sierra, M.I.; Valdés, A.; Fernandez, A.F.; Torrecillas, R.; Fraga, M.F. The effect of exposure to nanoparticles and nanomaterials on the mammalian epigenome. Int. J. Nanomed. 2016, 11, 6297-6306. [CrossRef]

28. Markou, A.; Zavridou, M.; Lianidou, E.S. miRNA-21 as a novel therapeutic target in lung cancer. Lung Cancer 2016, 7, 19-27. [PubMed]

29. Zhao, M.Y.; Wang, L.M.; Liu, J.; Huang, X.; Liu, J.; Zhang, Y.F. MiR-21 suppresses anoikis through targeting PDCD4 and PTEN in human esophageal adenocarcinoma. Curr. Med. Sci. 2018, 38, 245-251. [CrossRef] [PubMed]

30. Bica-Pop, C.; Cojocneanu-Petric, R.; Magdo, L.; Raduly, L.; Gulei, D.; Berindan-Neagoe, I. Overview upon miR-21 in lung cancer: Focus on NSCLC. Cell. Mol. Life Sci. 2018, 75, 3539-3551. [CrossRef]

31. Cao, M.; Seike, M.; Soeno, C.; Mizutani, H.; Kitamura, K.; Minegishi, Y.; Noro, R.; Yoshimura, A.; Cai, L.; Gemma, A. MiR-23a regulates TGF- $\beta$-induced epithelial-mesenchymal transition by targeting E-cadherin in lung cancer cells. Int. J. Oncol. 2012, 41, 869-875. [CrossRef] [PubMed] 
32. Kim, J.; Kim, T.Y.; Lee, M.S.; Mun, J.Y.; Ihm, C.; Kim, S.A. Exosome cargo reflects TGF- $\beta 1$-mediated epithelial-to-mesenchymal transition (EMT) status in A549 human lung adenocarcinoma cells. Biochem. Biophys. Res. Commun. 2016, 478, 643-648. [CrossRef]

33. Karacosta, L.G.; Anchang, B.; Ignatiadis, N.; Kimmey, S.C.; Benson, J.A.; Shrager, J.B.; Tibshirani, R.; Bendall, S.C.; Plevritis, S.K. Mapping lung cancer epithelial-mesenchymal transition states and trajectories with single-cell resolution. Nat. Commun. 2019, 10, 5587. [CrossRef]

34. Li, J.; Li, P.; Chen, T.; Gao, G.; Chen, X.; Du, Y.; Gao, F.; Zhang, G. Expression of microRNA-96 and its potential functions by targeting FOXO3 in non-small cell lung cancer. Tumor Biol. 2015, 36, 685-692. [CrossRef] [PubMed]

35. Schaaij-Visser, T.B.; de Wit, M.; Lam, S.W.; Jiménez, C.R. The cancer secretome, current status and opportunities in the lung, breast and colorectal cancer context. Biochim. Biophys. Acta 2013, 1834, 2242-2258. [CrossRef] [PubMed]

36. Ren, J.; Li, X.; Dong, H.; Suo, L.; Zhang, J.; Zhang, L.; Zhang, J. miR2103p regulates the proliferation and apoptosis of nonsmall cell lung cancer cells by targeting SIN3A. Exp. Ther. Med. 2019, 18, 2565-2573. [PubMed]

37. Guo, H.; Li, Q.; Li, W.; Zheng, T.; Zhao, S.; Liu, Z. miR-96 downregulates RECK to promote growth and motility of non-small cell lung cancer cells. Mol. Cell. Biochem. 2014, 390, 155-160. [CrossRef] [PubMed]

38. Liu, B.; Sun, X. miR-25 promotes invasion of human non-small cell lung cancer via CDH1. Bioengineered 2019, 10, $271-281$. [CrossRef]

39. Wu, H.; Zhou, J.; Mei, S.; Wu, D.; Mu, Z.; Chen, B.; Xie, Y.; Ye, Y.; Liu, J. Circulating exosomal microRNA-96 promotes cell proliferation, migration and drug resistance by targeting LMO7. J. Cell. Mol. Med. 2017, 21, 1228-1236. [CrossRef] [PubMed]

40. Svoronos, A.A.; Engelman, D.M.; Slack, F.J. OncomiR or tumor suppressor? The duplicity of microRNAs in cancer. Cancer Res. 2016, 76, 3666-3670. [CrossRef] [PubMed]

41. Alizadeh-Fanalou, S.; Khosravi, M.; Alian, F.; Rokhsartalb-Azar, S.; Nazarizadeh, A.; Karimi-Dehkordi, M.; Mohammadi, F. Dual role of microRNA-1297 in the suppression and progression of human malignancies. Biomed. Pharmacother. 2021, 141, 111863. [CrossRef]

42. Li, M.; Huo, X.; Davuljigari, C.B.; Dai, Q.; Xu, X. MicroRNAs and their role in environmental chemical carcinogenesis. Environ. Geochem. Health 2019, 41, 225-247. [CrossRef] [PubMed]

43. Ferrante, M.; Cristaldi, A.; Oliveri, C.G. Oncogenic role of miRNA in environmental exposure to plasticizers: A systematic review. J. Pers. Med. 2021, 11, 500. [CrossRef] [PubMed] 